Reprod. Nutr. Dévelop., 1987, 27 (1 B), 201-202.

\title{
Influence de la nature de l'aliment concentré complémentaire sur l'encombrement des rations par la chèvre en lactation
}

\author{
Sylvie GIGER, D. SAUVANT, J. HERVIEU
}

Station de Nutrition et Alimentation (I.N.R.A.) de I'I.N.A.-P.G. 16, rue Claude Bernard, 75231 Paris Cedex 05.

Summary. The main factors of variation of dry matter intake (DMI) of mixed diets given to dairy goats are milk production and concentrate level. This study points out that the kind of concentrate, for a given level of organic matter digestibility, had little individual effect on the DMl since the goats ate about $125 \mathrm{~g}$ /day more with a blend rich in high digestible fibre than with a cereal blend, which represented about $9 \%$ of forage intake.

Dans le système français des unités d'encombrement (UE), appliqué aux vaches laitières, la valeur UE de l'aliment concentré ne dépend que de celle du fourrage (Demarquilly et al., 1978), alors qu'elle est constante dans d'autres systèmes d'unité d'encombrement (Hyppola et Hasunen, 1970 ; Kristensen, 1983). Or, des résultats expérimentaux récents, obtenus sur caprins (Sauvant et al., 1980), ovins (Berge et Dulphy, 1985) et vaches laitières (Vérité et Dulphy, 1981) montrent que la nature de l'aliment concentré influence son taux de substitution. L'étude présentée a pour objet de comparer, sur la base des résultats regroupant 6 expérimentations, l'influence de la nature de l'aliment concentré, sur l'ingestion des rations offertes à la chèvre en lactation.

Matériel et méthodes. Les 6 expériences considérées ont porté sur 42 chèvres multipares en phase descendante de lactation, de la $7^{e}$ à la $20^{\circ}$ semaine après la mise-bas, qui produisaient de 0,06 à $0,20 \mathrm{~kg}$ de lait à $3,5 \%$ de $\mathrm{TB} / \mathrm{kg} \mathrm{P} \mathrm{P}^{0,75}$. Les chèvres recevaient une ration à base de foin de luzerne (F) ou d'ensilage de maïs (E) ou une association de ces deux fourrages (F + E) (tabl. 1). Le taux de refus du fourrage est compris entre 10 et $35 \%$. Dans chaque expérimentation, deux mélanges concentrés ont été formulés, à partir des tables I.N.R.A. 1978, de manière à présenter la même digestibilité de la matière organique (DMO) et être iso-azotés. Pour l'un, la teneur en parois (NDF) a été minimisée (aliments riches en amidon : $A$, contenant environ $75 \%$ de céréales ou de leurs sous-produits) et pour l'autre, maximisée (aliments riches en paroi facilement digestible : $P$, soit environ $60 \%$ pour l'ensemble « téguments de soja, pulpes de betteraves et

TABL. 1. - Influence de la production laitière et de la quantité de MS/ de concentré et de la production laitière corrigée à $3,5 \%$ de TB (PL35) sur la quantité totale de MSI.

$N^{\circ}$ expérience

Ration de base ( $\left.{ }^{1}\right)$

Type de concentré $\left({ }^{2}\right)$

Nombre d'observations

Nombre de chèvres

MSI totale $\mathrm{g} / \mathrm{kg} P^{0,75}\left({ }^{3}\right)$

MSI concentré $\mathrm{g} / \mathrm{kg} \mathrm{P}^{0,75}$

PL35 g/kg $P^{0,75}$

\begin{tabular}{|c|c|c|c|c|c|c|c|c|c|c|c|}
\hline \multicolumn{2}{|c|}{1} & \multicolumn{2}{|c|}{2} & \multicolumn{2}{|c|}{${ }^{3} \mathrm{~F}$} & \multicolumn{2}{|c|}{4} & \multicolumn{2}{|c|}{5} & \multicolumn{2}{|c|}{$\begin{array}{l}6 \\
E\end{array}$} \\
\hline A & $P$ & A & $P$ & A & $P$ & A & $P$ & A & $P$ & A & $P$ \\
\hline 11 & 9 & 8 & 8 & 4 & 4 & 4 & 4 & 9 & 9 & 9 & 5 \\
\hline 3 & 3 & 4 & 4 & 4 & 4 & 4 & 4 & 3 & 3 & 3 & 3 \\
\hline 112 & 122 & 108 & 118 & 118 & 127 & 113 & 117 & 111 & 122 & 103 & 96 \\
\hline 73 & 65 & 29 & 28 & 51 & 56 & 49 & 45 & 58 & 56 & 42 & 37 \\
\hline 124 & 144 & 158 & 169 & 146 & 152 & 172 & 168 & 132 & 147 & 122 & 91 \\
\hline
\end{tabular}

(1) F : Foin de luzerne; E : Ensilage de mais ; ( $\left.{ }^{2}\right)$ A : Aliment riche en céréales et leurs sousproduits; P : aliment riche en paroi facilement digestible; ( $\left.{ }^{3}\right)$ Données corrigées par le modèle d'analyse de variance-covariance. 
d'agrumes 11 . Les 84 observations effectuées correspondent chacune à une semaine de mesures de bilans digestifs, de 5 jours consécutifs, avec, en moyenne, deux périodes de mesure par chèvre. Le concentré a été distribué à raison de $350 \mathrm{~g} \mathrm{MS} / \mathrm{kg}$ de lait au-dessus des besoins couverts par la ration de base.

Afin de tenir compte des principaux facteurs de variation liés à la capacité d'ingestion de l'animal, les différentes quantités sont exprimées par rapport au poids métabolique $\left(\mathrm{P}^{0,75}\right)$ : matière sèche ingérée totale (MSI) ou d'aliment concentré $(Q$ conc.) et production laitière à $3,5 \%$ de taux butyreux (PL35). L'introduction de ces deux dernières variables comme facteur explicatif de la MSI permet de comparer les ingestions des fourrages à niveau égal d'apport de concentré et de production laitière. Les effets liés au fourrage $(6$ associations fourrageconcentré), au type de concentré (A ou P) et à l'interaction fourrage-concentré ont été testés par rapport à l'effet chèvre intra-régimes dans la variation résiduelle de l'équation reliant la quantité totale de matière sèche ingérée aux quantités de lait et de concentré.

Résultats et discussion. La quantité de matière sèche ingérée (MSI) a été en moyenne de $115,0 \mathrm{~g} / \mathrm{kg} \mathrm{P}^{0,75}$, avec des extrêmes de 65 et $165 \mathrm{~g} / \mathrm{kg} \mathrm{P}^{0,75}$. Les valeurs de digestibilité in vivo de la matière organique ne diffèrent pas entre les régimes $A$ et $P$, à même niveau d'apport de concentré. La production laitière ( $P L$ 35) permet d'expliquer les deux tiers des variations de la matière sèche totale ingérée :

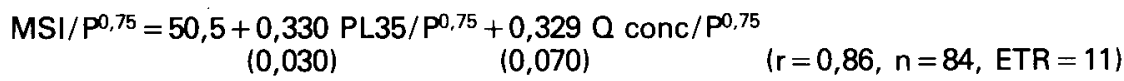

La quantité de MSI augmente, en moyenne, de $330 \mathrm{~g}$ par $\mathrm{kg}$ de lait et par $\mathrm{kg}$ de concentré. L'analyse de variance indique que le test d'interaction n'est pas significatif ; les effets fourrage et concentré peuvent donc être considérés comme additifs. Seul l'effet concentré est significatif au seuil de 7,5\%. A production laitière et apport de concentré égaux, les chèvres qui reçoivent le concentré riche en paroi ingèrent en moyenne $6,26 \mathrm{~g} \mathrm{MS} / \mathrm{kg} \mathrm{P}^{0,75}$ (soit environ $135 \mathrm{~g} \mathrm{MS} /$ animal) de matière sèche totale en plus que celles qui reçoivent l'aliment $A$ qui a la même digestibilité que le précédent. Cette différence représente en moyenne $10 \%$ de la matière sèche du fourrage ingéré. Elle pourrait donc être causée par une interaction digestive, puisque la quantité du fourrage ingérée diminue dans le cas où l'aliment concentré apporte des quantités importantes d'amidon. Ce résultat montre l'influence de la nature de l'aliment concentré, lorsqu'il est donné en proportions importantes (environ $\mathbf{4 0} \%$ de la MS), sur l'ingestion de la ration et confirme les résultats précédents : a DMO égale, le coefficient de substitution le plus élevé correspond à l'aliment de type amidon (Sauvant et al., 1980). II est également à rapprocher des résultats de Berge et Dulphy (1985) montrant que la pulpe de betteraves est moins encombrante que l'orge ou le son de blé. Il paraît donc souhaitable de pouvoir intégrer la qualité du concentré dans le système des unités d'encombrement.

Berge Ph., Dulphy J. P., 1985. Ann. Zootech., 34, 313-334.

Demarquilly C.., Andrieu J., Sauvant D., 1978. 459-518. In Alimentation des ruminants (Ed. R. Jarrige), I.N.R.A. Publications, route de St Cyr, 78000 Versailles.

Hyppola K., Hasunen O., 1970. Acta agr. fenn., 116, 1-59.

Kristensen E. S., 1983. Beret. Statens Husdyrbrugsfors, 52, 78-109.

Sauvant D., Giger S., Morand-Fehr P., 1980. 310 réunion annuelle de la F.E.Z., Munich.

Vérité R., Dulphy J. P., 1981. Bull. Tech. C.R.Z.V. Theix I.N.R.A., 45, 15-21. 